\title{
Making the Most of Kepler Photometry: Characterizing Exoplanets through Phase Curve Analysis
}

\author{
Lisa J. Esteves ${ }^{1, \text { a }}$, Ray Jayawardhana ${ }^{2}$, and Ernst de Mooij ${ }^{1}$ \\ 1 Astronomy \& Astrophysics, University of Toronto, Toronto, Canada \\ 2 Physics \& Astronomy, York University, Toronto, Canada (Presenting Author)
}

\begin{abstract}
The Kepler mission's long-term monitoring of stars through high-precision photometry has not only revealed a plethora of exoplanet transits but also provided valuable data for characterizing a subset of these planets. Using over four years of Kepler observations, we have derived phase curves for over a dozen planets, and use these measurements to constrain their mass, brightness/temperature and energy redistribution between the day and the night sides. In our new study, we also investigate possible offsets of the peak brightness of the phase curve, which could be indicative of inhomogeneous clouds and/or substantial winds in the planet's atmosphere. We find significant offsets for over a half-dozen planets. With this growing sample of measured phase curves, we are able to better examine the trends of hot Jupiter energy budgets and albedos, and for the first time relate these properties to the presence of clouds or winds on a planet.
\end{abstract}

\section{Introduction}

The main goal of the Kepler mission was to find planets using the transit method, the high precision, long baseline and continuous nature of its observations make the resulting photometry ideal for characterizing exoplanets through studies of their phase variations. From transit measurements, it is possible to derive both the planet's orbital parameters as well as the planet-to-star radius ratio. Meanwhile, the out-of-transit light curve, known as the phase curve, can yield constraints on the planetary atmosphere (e.g. albedo, brightness temperature) and the planet's mass.

For a number of planets, Spitzer phase curve observations have provided direct measurements of the day-night contrasts and have shown that often the hottest spot in the planet's atmosphere is offset from the sub-stellar point due to super-rotating jets (e.g. Knutson et al. 2007). While Demory et al. (2013) have shown, using Kepler phase curve observations and Spitzer secondary eclipse measurements, that Kepler-7b is dominated by reflected light in the optical and inhomogeneous clouds cause albedo variations between the morning and evening sides of the planet.

\section{Our Analysis}

Here we present the results of our transit and phase curve analysis (Esteves et al. 2014), with the inclusion of a phase function offset, for 14 planets (Kepler-5b, Kepler-6b, Kepler-7b, Kepler-8b, Kepler10b, Kepler-12b, Kepler-41b, Kepler-43b, Kepler-76b, Kepler-91b, Kepler-412b, KOI-13b, TrES-2b and HAT-P-7b) using all 18 quarters of Kepler's long-cadence and short-cadence data.

After the removal of systematics and outliers (see Esteves et al. 2013 for details), the phase curves were fit with several models, which varied in the number of free parameters including the planet's mass, eclipse depth, phase function amplitude and peak offset. Best fit models were chosen using

\footnotetext{
a e-mail: esteves@astro.utoronto.ca
}

This is an Open Access article distributed under the terms of the Creative Commons Attribution License 4.0, which permits unrestricted use, distribution, and reproduction in any medium, provided the original work is properly cited. 

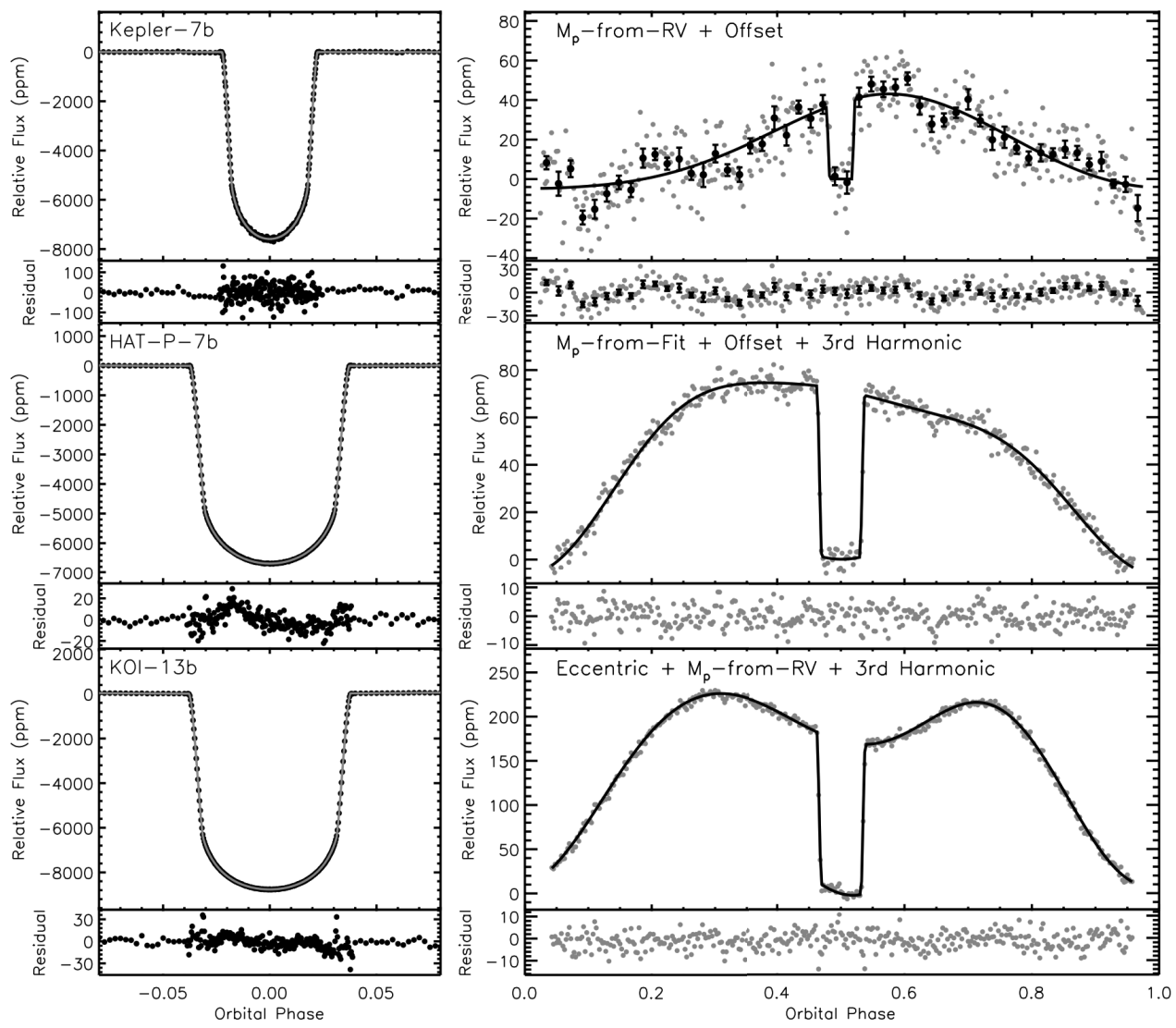

Fig. 1. The left and right panels contain the binned and phase-folded transit light curves and phase curves, respectively, of select planets in our sample. Top: Kepler-7b, which exhibits an eastward offset (i.e. a shift in peak planetary brightness shift to after mid-eclipse). Middle: HAT-P-7b, which exhibits a westward offset (i.e. a shift in peak planetary brightness shift to before mid-eclipse). Bottom: KOI-13b, where we find an additional sinusoidal signal at one third the planet's period.

the Bayesian Information Criterion and parameters were estimated using Markov Chain Monte Carlo simulations. Three of the fourteen fits can be found in Figure 1.

\section{Results}

From our analysis of phase variations in fourteen Kepler planets, we find that most have low geometric albedos $<0.25$ dominated by reflected light, with the exception of Kepler-10b, Kepler-91b and KOI$13 \mathrm{~b}$ where we derive values of $0.58,0.39$ and 0.46 , respectively. Of our targets, KOI-13b, with a small eccentricity of $6.4_{-1.6}^{+1.2} \times 10^{-4}$, is the only planet where an eccentric orbit is favoured.

For KOI-13b, we again detect a third cosine harmonic with an amplitude of $7.0 \pm 0.3 \mathrm{ppm}$ and for HAT-P-7b we detect a never-before-seen third harmonic with an amplitude of $1.9 \pm 0.2 \mathrm{ppm}$. The cause of this additional third harmonic could be the movement of the stellar tidal bulge raised by the planet, the source of ellipsoidal variations, across areas of the star with different surface brightnesses. The motivation for this reasoning is the asymmetry in KOI-13b's and HAT-P-7b's transit, where the former is caused by a spin-orbit misalignment and significant gravity darkening due to rapid stellar rotation (Szabo et al. 2011; Barnes et al. 2011).

For seven planets (Kepler-5b, Kepler-6b, Kepler-10b, Kepler-91b, Kepler-412b, KOI-13b and TrES$2 b)$ our analysis did not favour an offset in the peak of their planetary light, while for the other seven 


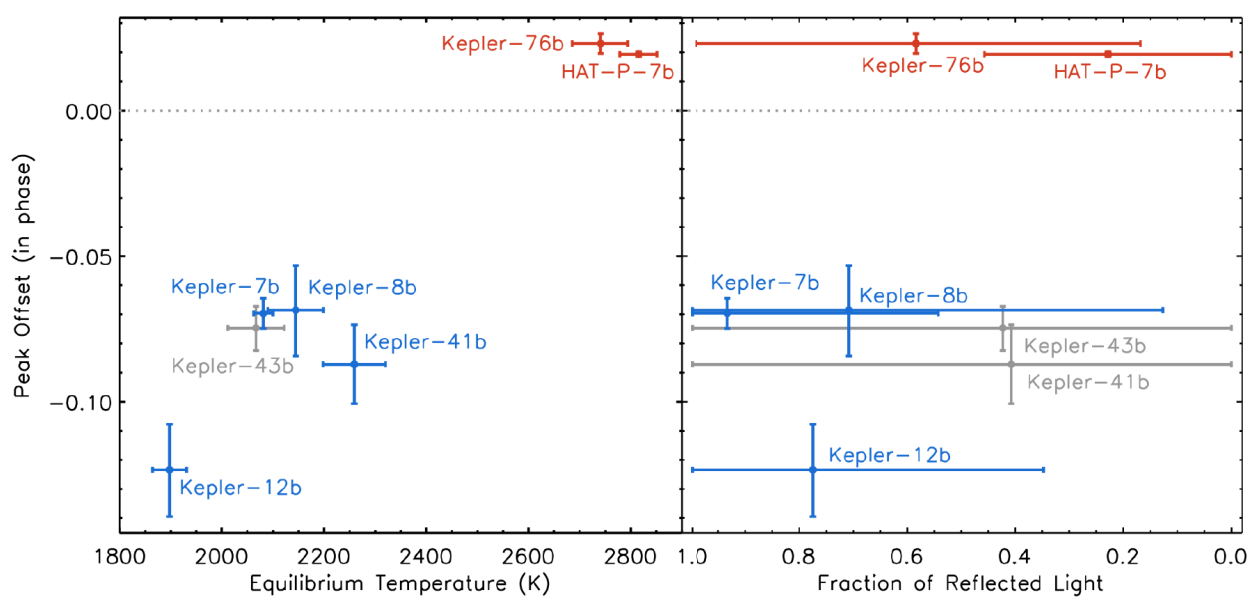

Fig. 2. Left: Peak offset of the planet's phase function vs. maximum equilibrium temperature. Right: Peak offset of the planet's phase function vs. the fraction of the planet's brightness due to reflected light. Blue points indicate an eastward offset, red points indicate a westward offset and the grey points indicate values that are either completely unconstrained, are in doubt due to light curve variability or are strongly influenced by the chosen stellar parameters (KOI-13b). Dotted grey line indicates the boundary between eastward and westward shifts.

(Kepler-7b, Kepler-8b, Kepler-12b, Kepler-41b, Kepler-43b, Kepler-76b and HAT-P-7b) we find both eastward and westward offsets. When we compare our measured peak offsets to the planet's equilibrium temperature (see left panel of Figure 2) we see a division between planets around an equilibrium temperature of approximately $2500 \mathrm{~K}$, where hotter planets have a positive or eastward shift and cooler planets have a negative or westward shift.

The self-consistent albedo measurements give strong evidence that, for most planets, the light from the planet in the Kepler band is a combination of both reflected light and thermal emission. Since Kepler observes in a single broad optical band, it is difficult to constrain the relative contributions from each source. However, using our self-consistent albedo equation we can estimate the reflected light fraction by dividing the observed geometric albedo by our self-consistent albedo. For the seven planets with peak offsets of their planetary light, we compare the reflected light fraction and peak offset in the right panel of Figure 2. For the three planets with a westward offset and a constraint on their reflected light fraction, it appears that at least some reflectivity is needed explain their observed brightnesses.

\section{Interpretation}

For tidally locked hot-Jupiters, with a thermal emission dominated brightness, a robust outcome of a variety of circulation models (e.g. Cooper et al. 2005; Heng et al. 2011) combined with analytical theory Showman et al. (2011) is an eastward shift of the planet's peak brightness. On the other hand, Demory et al. (2013) use the lack of significant thermal emission in the Spitzer 3.6 and $4.5 \mu \mathrm{m}$ bandpasses and the presence of a westward shift to conclude that Kepler-7b's phase curve is dominated by reflected light. Furthermore they state that the most likely cause of the westward shift is the presence of inhomogeneous reflective clouds, whose properties change as a function of longitude and are influenced by the planet's wind and thermal patterns.

With Kepler alone we are not able to determine whether planets with a peak offset are dominated by reflected light or thermal emission. However, if an equilibrium temperature of approximately $2500 \mathrm{~K}$ is an upper boundary on cloud formation (e.g. Fortney et al. 2008; Morley et al. 2013), the phase functions of Kepler-76b and HAT-P-7b would be dominated by thermal emission and therefore our measurement of an eastward shift is consistent with circulation models of super-rotating jets. On the 
other hand, if the planets under $2500 \mathrm{~K}$ are able to condense particles, it is possible that their phase functions and westward shifts are caused by inhomogeneous reflective clouds as seen in Kepler-7b (Demory et al. 2013).

For tidally locked planets, a westward offset (or a peak after eclipse) means that the planet appears brightest on the morning-side. If this morning-side brightness is produced by reflective clouds, it is conceivable that these clouds could condense on the night-side, when temperatures are cooler, and then dissipate as the atmosphere heats up during the day, thus resulting in thicker, more reflective clouds at sunrise that gradually disperse throughout the day.

\section{Conclusions}

These results have drastically increased the number of Kepler planets with detected planetary light offsets and provided the first evidence, in the Kepler data, for a correlation between the direction of the peak offset and the planet's temperature. This correlation could possibly arise if hotter planets are dominated by thermal emission and therefore exhibit a hot spot shifted to the east, as theoretically predicted, whereas cooler planets are dominated by reflected light and have clouds westward of the substellar point (i.e. on the morning-side), as seen for Kepler-7b. However, with this study alone we are not able to determine whether the planets with peak offsets are seen predominantly in reflected light or thermal emission.

\section{References}

1. Barnes et al. ApJS 197, (2011) 10

2. Cooper \& Showman ApJL 629 (2005) L45

3. Demory et al. ApJL 776 (2013) L25

4. Esteves et al. ApJ 772 (2013) 51

5. Esteves et al. Arxiv Preprint (2014) arXiv:1407.2245

6. Fortney et al. ApJ 678 (2008) 1419

7. Heng et al. MNRAS 413 (2011) 2380

8. Knutson et al. Nature 447 (2007) 183

9. Morley et al. ApJ 775 (2013) 33

10. Szabó et al. ApJL 736 (2011) L4 